\title{
Clauser-Horne inequality and decoherence in mesoscopic conductors
}

\author{
Elsa Prada \\ Departamento de Física Teórica de la Materia Condensada, \\ C-V, Universidad Autónoma de Madrid, E-28049 Madrid, Spain \\ Institut für Theoretische Festkörperphysik, Universität Karlsruhe, D-76128 Karlsruhe, Germany \\ Fabio Taddei and Rosario Fazio \\ NEST-INFM \& Scuola Normale Superiore, I-56126 Pisa, Italy
}

(Dated: February 2, 2008)

\begin{abstract}
We analyze the effect of decoherence on the violation of the Clauser-Horne $(\mathrm{CH})$ inequality for the full electron counting statistics in a mesoscopic multiterminal conductor. Our setup consists of an entangler that emits a flux of entangled electrons into two conductors characterized by a scattering matrix and subject to decoherence. Loss of phase memory is modeled phenomenologically by introducing fictitious extra leads. The outgoing electrons are detected using spin-sensitive electron counters. Given a certain average number of incoming entangled electrons, the CH inequality is evaluated as a function of the numbers of detected particles and on the various quantities characterizing the scattering matrix. When decoherence is turned on, we show that the amount of violation of the $\mathrm{CH}$ inequality is effectively reduced. Interestingly we find that, by adjusting the parameters of the system, there exists a protected region of $Q$ values for which violation holds for arbitrary strong decoherence.

PACS numbers: 03.65.Ud, 03.65.Yz, 72.70.+m, 72.90.+y
\end{abstract}

\section{INTRODUCTION}

Entanglement [1] is probably the most important resource for the implementation of quantum computation and quantum communication protocols [2]. Since recently, most of the work on entanglement has been carried out in optical systems using photons 3], cavity QED systems [4], and ion traps [5]. Solid state systems, however, are a very attracting arena of research in quantum information [6, 7, 8] because, in perspective of future applications, they should allow for scalability and integration. In this light, a number of different realizations of entangled electrons have since been proposed: hybrid normal-superconducting structures [9, 10, 11, 12, 13, 14, 15, 16], superconductorcarbon nanotubes systems [17, 18, 19], quantum dots in the Coulomb blockade regime [6, 20, 21], chaotic quantum dots 22], Kondo-like impurities [23], quantum Hall bar systems 24, 25, 26, 27, 28], Coulomb scattering in $2 \mathrm{D}$ electron gas [29].

Besides its generation, a crucial issue is that of the detection of entanglement. By means of a beam-splitter, entanglement can be detected in transport through an analysis of current noise [30] or higher cumulants [31]. Furthermore, the presence of entanglement can be revealed by analyzing the Bell inequality and quantities like concurrence [32], which have been expressed in terms of zero-frequency charge and spin-current noise 10, 11, 12, 24, 27, 33, 34]. Violation of a Bell inequality implies that there exist quantum correlations between the detected particles that cannot be described by any local hidden variables theory. In the same spirit as it was done for the noise, in Ref. 35 a Clauser-Horne $(\mathrm{CH})$ inequality [37, 38] was derived for the Full Counting Statistics
(FCS) of electrons and its properties were discussed [39]. In particular, it was found that the maximum violation of the $\mathrm{CH}$ inequality for electrons in the Bell state simply scales as the inverse of the number of injected particles. It was also found that the $\mathrm{CH}$ inequality is violated for a superconducting hybrid structure and, more interestingly, for a three terminal fully normal device.

In real systems electrons are unavoidably coupled to the electromagnetic environment. As a result dephasing takes place, thereby reducing and eventually destroying entanglement. Understanding the consequences of dephasing is an important issue. In Refs. 12, 15, 25, 40 the effect of dephasing was mimicked by introducing in the density matrix of the electronic entangled states a phenomenological parameter which suppresses its offdiagonal elements. By properly choosing the transmission probability of beam-splitters or tunnel barriers, violation of Bell inequality was found even for "strong" dephasing. In Refs. 24, 41 dephasing was introduced averaging over an uniform distribution of random phase factors accumulated in each edge channel of the quantum Hall bar. If the two edge channels are mixed by the tunnel barrier, no violation was reported for "strong" dephasing. The effect of decoherence and relaxation has also been analyzed using a Bloch equation formalism in Ref. 42.

In the present work we analyze the $\mathrm{CH}$ inequality for the FCS [35] in the presence of dephasing. We consider the prototype setup depicted in Fig. 11 consisting of a generic entangler connected to two conducting wires. Entangled electrons injected in the two leads are detected by performing spin-selective counting along a given local quantization axis. The entangled electrons are subject to decoherence while transversing the conductors (thus 
before reaching the detectors) 36. Various phenomenological methods have been developed to treat dephasing in transport through mesoscopic conductors. In Refs. 43, 44, which actually describes exactly nonequilibrium radiation acting on the system, dephasing is induced by a classical fluctuating potential. In Ref. 45, dephasing is treated as random fluctuations of the phase of propagating modes through the conductor. Both methods have been recently applied to FCS in Refs. 46, 47. In this paper decoherence is introduced as due to the presence of additional fictitious reservoirs along both wires. This method, which mimics the effect of inelastic processes, was introduced by Büttiker [48, 49] in terms of fictitious extra leads [50]. The advantage of this model resides in the fact that inelastic, phase randomization processes are implemented within an elastic, time-independent scattering problem. In the rest of the paper we shall refer to decoherence as to the effect produced by such fictitious additional leads.

As expected, we find that decoherence suppresses the violation of the $\mathrm{CH}$ inequality, though leaving unchanged the range of angles for which violation occurs. In particular, the value of the maximum violation is suppressed more rapidly as compared with the absence of decoherence (exponentially with the square root of the number of injected electrons instead of algebraically). Importantly, by studying the $\mathrm{CH}$ inequality as a function of the number of transmitted electrons, there exist values of such quantity that are more protected against decoherence.

The paper is organized as follows: In Section [II we described in detail the mesoscopic system we are considering to test the violation of the $\mathrm{CH}$ inequality together with the phenomenological model of decoherence. Section [II is devoted to the formulation of the $\mathrm{CH}$ inequality for the FCS within the scattering approach and to the analysis of the no-enhancement assumption (Section III. The results are presented in Section IV where a systematic analysis of the violation of the $\mathrm{CH}$ inequality against all the parameters of the device is addressed. A concluding summary is provided in Section $\nabla$

For completeness, we include in Appendix $\$$ the results relative to an asymmetric setup, whereby decoherence occurs only in one of the two wires. In Appendix B and C we collect, respectively, the expressions of the expectation values and the different probability distributions.

\section{DESCRIPTION OF THE SYSTEM}

We consider the setup illustrated in Fig. 1. It consists of an entangler, two conducting wires and two spinselective counters. The entangler, on the left-hand-side, is a device that produces pairs of electrons, with energy $E<\mu_{\mathrm{L}}$, in a maximally spin entangled state (Bell state). On the right-hand-side of Fig. 1 the electron counting is performed in leads 1 and 2 (at equal electrochemical potential $\mu_{\mathrm{R}}$ ) for electrons with spin aligned along the local

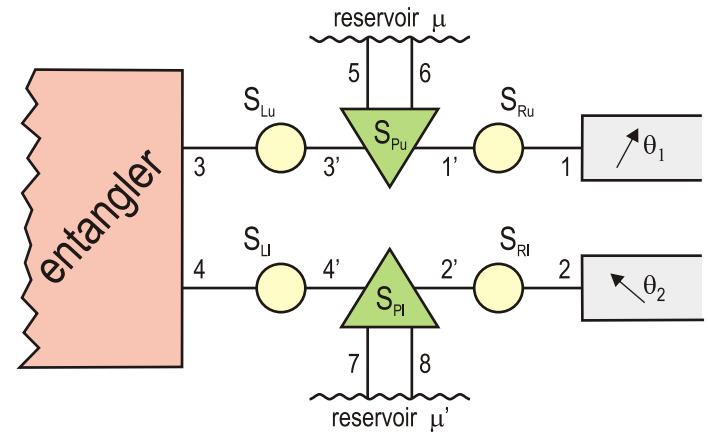

FIG. 1: (Color online) Idealized setup for testing the $\mathrm{CH}$ inequality for electrons in a solid-state environment in the presence of decoherence. It consists of three parts: An entangler that produces pairs of spin-entangled electrons exiting from terminals 3 and 4 . Two conductors that connect these terminals with exiting leads 1 and 2, and two analyzers. The conductors are described by the elastic scattering matrices $S_{\mathrm{Lu}}, S_{\mathrm{Ru}}, S_{\mathrm{Ll}}$ and $S_{\mathrm{Rl}}$, and the inelastic ones $S_{\mathrm{Pu}}$ and $S_{\mathrm{Pl}}$. These last ones can simulate phenomenologically the presence of decoherence through the coupling via two leads $(5,6$ and $7,8)$ to two additional reservoirs of chemical potentials $\mu$ and $\mu^{\prime}$. Electron counting is performed in leads 1 and 2. Finally, $\theta_{1}$ and $\theta_{2}$ are the angles at which the spin-quantization axis are oriented.

spin-quantization axis at angles $\theta_{1}$ and $\theta_{2}$ (spin-selective counters). As a convention we say that the analyzer is not present when the electron counting is spin-insensitive (electrons are counted irrespective of their spin direction). Since we assume no back-scattering from counters to the entangler, the particles which are not counted are lost and hence there is no communication between the two detectors. Leads 3 and 4 of the entangler are connected to exit leads 1 and 2 through two conductors, where inelastic processes are introduced through the fictitious lead model of Büttiker [52]. Let us analyze in detail the upper wire (see Fig. 1) which connects the emitting lead 3 with the exiting lead 1 . The conductor consists of three scattering regions. The elastic scatterer connecting lead 3 to 3 ' is described by the matrix

$$
\hat{S}_{\mathrm{Lu}}=\left(\begin{array}{cc}
\check{r} & \check{t}^{\prime} \\
\check{t} & \check{r}^{\prime}
\end{array}\right)=\left(\begin{array}{cccc}
r_{\uparrow} & 0 & t_{\uparrow}^{\prime} & 0 \\
0 & r_{\downarrow} & 0 & t_{\downarrow}^{\prime} \\
t_{\uparrow} & 0 & r_{\uparrow}^{\prime} & 0 \\
0 & t_{\downarrow} & 0 & r_{\downarrow}^{\prime}
\end{array}\right) .
$$

[The index L (R) stands for Left (Right) elastic scatterer, while P stands for Probe scatterer; u (l) refers to the upper (lower) wire.] Here $r_{\sigma}\left(t_{\sigma}\right)$ is the probability amplitude for an incoming particle with spin $\sigma$ from lead 3 to be reflected (transmitted into lead 3'). For a normal single-channel wire we set $t_{\sigma}=t_{\sigma}^{\prime}=\sqrt{T_{0}}$, and $r_{\sigma}=r_{\sigma}^{\prime}=i \sqrt{1-T_{0}}$, where $T_{0}$ is the transmission probability. Inelastic scattering is introduced by plugging in an additional reservoir of chemical potential $\mu$ with an 
energy- and spin-independent scattering matrix

$$
\bar{S}_{\mathrm{Pu}}=\left(\begin{array}{cccc}
\check{0} & \sqrt{1-\alpha} \check{1} & \sqrt{\alpha} \check{1} & \check{0} \\
\sqrt{1-\alpha} \check{1} & \check{0} & \check{0} & \sqrt{\alpha} \check{1} \\
\sqrt{\alpha} \check{1} & \check{0} & \check{0} & -\sqrt{1-\alpha} \check{1} \\
\check{0} & \sqrt{\alpha} \check{1} & -\sqrt{1-\alpha} \check{1} & 0
\end{array}\right),
$$

represented by a triangle in Fig. 1] For the sake of clarity, we have denoted by a check $\left(^{\sim}\right)$, a caret $\left(^{\wedge}\right)$ and an overbar $\left(^{-}\right)$, respectively, $(2 \times 2),(4 \times 4)$ and $(8 \times 8)$ matrices. In Eq. (2) $\check{1}$ and 0 are, respectively, unit and zero $(2 \times 2)$-matrices in the spin space, and $(1-\alpha)$ is the probability for transmitting a particle between leads 3' and 1 '. The coupling parameter ranges from $\alpha=0$, when no particles are transmitted into leads 5 and 6 from leads 3 ' and 1', to $\alpha=1$, when no particles are transferred between leads 3' and 1'. A third elastic scatterer, described by a matrix $\hat{S}_{\mathrm{Ru}}$, connects lead 1' to lead 1 . The conductor is therefore described by the matrix $\hat{S}_{13}$ defined as $\hat{S}_{13}=\hat{S}_{\mathrm{Ru}} \otimes \bar{S}_{\mathrm{Pu}} \otimes \hat{S}_{\mathrm{Lu}}$, where the notation $\otimes$ stands for the scattering matrix composition (elimination of internal current amplitudes) [53]. For simplicity, we shall assume that $\hat{S}_{\mathrm{Ru}}=\hat{S}_{\mathrm{Lu}}$.

Due to the presence of the additional reservoir, particles propagating through lead 3 are transmitted partially to lead 1 and partially to leads 5 and 6 (see Fig. 1). The additional reservoir, however, can transfer itself particles to lead 1 and 3. As a result, only a fraction of the particles arriving in lead 1 comes from coherently transmitted ones sent in from lead 3, with probability

$$
T_{13}=\frac{T_{0}^{2}(1-\alpha)}{\left[1+\left(1-T_{0}\right)(1-\alpha)\right]^{2}}
$$

Another fraction, the incoherent contribution, comes from the additional reservoir through leads 5 and 6 , with probability

$$
T_{15}+T_{16}=\frac{T_{0} \alpha}{1+\left(1-T_{0}\right)(1-\alpha)} .
$$

The presence of the extra reservoir mimics the fact that the current flowing through the conductor is partially composed of particles (the incoherent fraction) which have lost phase memory while traversing it. For $\alpha=0$ all particles are coherently transmitted and $T_{15}+T_{16}=0$, while for $\alpha=1$ all particles are transferred incoherently and $T_{13}=0$. For $\alpha=0$, the overall transmission probability through the conductors is given by $T=T_{0}^{2} /\left(2-T_{0}\right)^{2}$. In the rest of the paper we will refer to $\alpha$ as to the decoherence rate.

The chemical potential $\mu$ of the additional reservoir is set in such a way that no net current flows in or out of the reservoir $\left(I_{5}+I_{6}=0\right)$. This constraint is enforced only on average. An instantaneous current in or out the additional reservoir is then allowed [48, 49, 54, 55], and a non-fluctuating chemical potential $\mu$ is assumed (for this reason the additional terminal does not behave as a voltage probe).
A similar description applies to the lower wire connecting lead 4 with lead 2 , so that the scattering matrix of the conductor is defined as $\hat{S}_{24}=\hat{S}_{\mathrm{Rl}} \otimes \bar{S}_{\mathrm{Pl}} \otimes \hat{S}_{\mathrm{Ll}}$, where, for simplicity, we set $\hat{S}_{\mathrm{Rl}}=\hat{S}_{\mathrm{Ll}}$. If the angles $\theta_{1}$ and $\theta_{2}$ of the analyzers are parallel to each other and in the absence of spin mixing processes, the total matrix of the system can be written as

$$
\bar{S}=\left(\begin{array}{cc}
\hat{S}_{13} & \hat{0} \\
\hat{0} & \hat{S}_{24}
\end{array}\right)
$$

The general scattering matrix relative to non-collinear angles $\bar{S}_{\theta_{1}, \theta_{2}}$ is obtained from $\bar{S}$ by rotating the spin quantization axis independently in the two conductors (note that this is possible because the two wires are decoupled) [56]: $\bar{S}_{\theta_{1}, \theta_{2}}=\overline{\mathcal{U}} \bar{S} \overline{\mathcal{U}}^{\dagger}$, where $\overline{\mathcal{U}}$ is the rotation matrix given by

$$
\overline{\mathcal{U}}=\left(\begin{array}{cccc}
\check{1} & \check{0} & \check{0} & \check{0} \\
\check{0} & \check{U}_{\theta_{1}} & \check{0} & \check{0} \\
\check{0} & \check{0} & \check{1} & \check{0} \\
\check{0} & \check{0} & \check{0} & \check{U}_{\theta_{2}}
\end{array}\right)
$$

and

$$
\check{U}_{\theta}=\left(\begin{array}{cc}
\cos \frac{\theta}{2} & \sin \frac{\theta}{2} \\
-\sin \frac{\theta}{2} & \cos \frac{\theta}{2}
\end{array}\right) .
$$

For simplicity, we further assume that the two conductors are equal and that they are subjected to the same degree of decoherence, so that $\hat{S}_{13}=\hat{S}_{24}$. For this reason the chemical potentials of the additional reservoirs are identical. It is interesting to notice that decoherence processes in the two wires are, in some sense, "uncorrelated", meaning that we have imposed that the currents flowing through the fictitious leads vanish separately in the two reservoirs. (Correlations can be introduced, for example, by imposing $I_{5}+I_{6}+I_{7}+I_{8}=0$.) In the symmetrical setup we are considering here, $\mu=\mu_{\mathrm{R}}+\left(\mu_{\mathrm{L}}-\mu_{\mathrm{R}}\right) / 2$. In the rest of the paper we consider the case in which all the reservoirs are at zero temperature.

The incoming state of the system $|\psi\rangle$ depends on whether the energy of electrons falls within the range $\mu_{\mathrm{R}}<E<\mu$ or $\mu<E<\mu_{\mathrm{L}}$ :

$$
|\psi\rangle=\left\{\begin{array}{ll}
\left|\psi_{\mathrm{B}}\right\rangle & \mu<E<\mu_{\mathrm{L}} \\
\left|\psi_{\mathrm{S}}\right\rangle & \mu_{\mathrm{R}}<E<\mu
\end{array},\right.
$$

where

$$
\left|\psi_{\mathrm{B}}\right\rangle=\frac{1}{\sqrt{2}}\left[a_{3 \uparrow}^{\dagger}(E) a_{4 \downarrow}^{\dagger}(E) \pm a_{3 \downarrow}^{\dagger}(E) a_{4 \uparrow}^{\dagger}(E)\right]|0\rangle,
$$

and

$$
\begin{aligned}
\left|\psi_{\mathrm{S}}\right\rangle & =\frac{1}{\sqrt{2}}\left[a_{3 \uparrow}^{\dagger}(E) a_{4 \downarrow}^{\dagger}(E) \pm a_{3 \downarrow}^{\dagger}(E) a_{4 \uparrow}^{\dagger}(E)\right] \\
& \times \prod_{n=5,6,7,8} a_{n \uparrow}^{\dagger}(E) a_{n \downarrow}^{\dagger}(E)|0\rangle .
\end{aligned}
$$


In Eqs. (9) and (10) $a_{i \sigma}^{\dagger}(E)$ is the creation operator for a propagating electron in lead $i$ with spin $\sigma$ at energy $E$. The upper sign refers to the case in which the incoming state is a spin triplet and the lower sign to the spin singlet. Electrons with energy between $\mu$ and $\mu_{\mathrm{L}}$ are exiting leads 3 and 4 of the entangler in a superposition of spin $\uparrow$ and $\downarrow$ states. For energies between $\mu_{\mathrm{R}}$ and $\mu$ electrons are also injected from the additional leads (with indexes $5,6,7$ and 8) in a factorized state. Note that this occurs only in the presence of decoherence, i.e. for $\alpha \neq 0$.

By setting $\mu_{\mathrm{R}}=0$ and $\mu_{\mathrm{L}}=e V$, the total current flowing in lead 1, calculated using the Landauer-Büttiker formalism [57] in the linear response regime, is given by

$$
I_{1}=e^{2} V / h\left(T_{13}+T_{15}+T_{16}\right)
$$

Although the coherent part of the current decreases with $\alpha$, the total current increases with it (except for $T=$ 1 , where it remains constant and equal to $\left.e^{2} V / h\right)$. We would like to mention that this is a special feature of the model of decoherence we are using, not to be expected in general.

\section{CH INEQUALITY FOR THE FULL COUNTING STATISTICS}

The quantity employed in the formulation of the $\mathrm{CH}$ inequality, as derived in Ref. 35 , is the joint probability $P\left(Q_{1}, Q_{2}\right)$ for transferring a number of $Q_{1}$ and $Q_{2}$ electronic charges into leads 1 and 2 over an observation time $t$. The $\mathrm{CH}$ inequality is based on the hypothesis that the outcome of a measurement could be accounted for by a local hidden variable theory. The test of the $\mathrm{CH}$ inequality proceeds as follows. The entangler is switched on during an observation time $t$ (where the minimum $t$ is the inverse of the measuring device bandwidth) in which it emits an average number $M$ of pairs of entangled electrons. After traversing the conductors (and being affected by inelastic scattering) the electrons are counted in both terminals 1 and 2. The experiment is then repeated to get single terminal and joint terminal probability distributions that $Q_{1}$ particles arrive into analyzer 1 and $Q_{2}$ particles arrive into analyzer 2 (along a local spin-quantization axis or independently of it) with $Q_{1}+Q_{2} \leq 2 M$.

The $\mathrm{CH}$ inequality for the FCS reads 35

$$
\begin{aligned}
\mathcal{S}_{\mathrm{CH}}= & P^{\theta_{1}, \theta_{2}}\left(Q_{1}, Q_{2}\right)-P^{\theta_{1}, \theta_{2}^{\prime}}\left(Q_{1}, Q_{2}\right)+P^{\theta_{1}^{\prime}, \theta_{2}}\left(Q_{1}, Q_{2}\right) \\
& +P^{\theta_{1}^{\prime}, \theta_{2}^{\prime}}\left(Q_{1}, Q_{2}\right)-P^{\theta_{1}^{\prime},-}\left(Q_{1}, Q_{2}\right) \\
& -P^{-, \theta_{2}}\left(Q_{1}, Q_{2}\right) \leq 0 .
\end{aligned}
$$

The possible violation, or the extent of it, also depends on $Q_{1}$ and $Q_{2}$. $P^{\theta_{1}, \theta_{2}}\left(Q_{1}, Q_{2}\right)$ is the joint probability in the presence of two analyzers, where $Q_{1}$ electrons are counted in lead 1 along $\theta_{1}$ direction and $Q_{2}$ are counted in lead 2 along $\theta_{2}$. $P^{\theta_{1},-}\left(Q_{1}, Q_{2}\right)$ is the corresponding joint probability when one of the two analyzers has been removed. The same notation will be used for single terminal probability distributions: $P^{\theta_{i}}\left(Q_{i}\right)$ in the presence of an analyzer and $P\left(Q_{i}\right)$ if no analyzer is present. Eq. (12) holds for all values of $Q_{1}$ and $Q_{2}$ which satisfy the noenhancement assumption:

$$
P^{\theta_{i}}\left(Q_{i}\right) \leq P\left(Q_{i}\right)
$$

The joint probability distribution for transferring $Q_{1 \sigma}$ electrons with spin $\sigma$ in lead $1, Q_{2 \sigma}$ electrons with spin $\sigma$ in lead 2 and so on is given by

$$
\begin{aligned}
& P\left(Q_{1 \uparrow}, Q_{1 \downarrow}, Q_{2 \uparrow}, \ldots\right) \\
& =\frac{1}{(2 \pi)^{2 n}} \int_{-\pi}^{+\pi} d \lambda_{1 \uparrow} d \lambda_{1 \downarrow} d \lambda_{2 \uparrow} \ldots \chi\left(\overrightarrow{\lambda_{\uparrow}}, \overrightarrow{\lambda_{\downarrow}}\right) \\
& \times e^{i \overrightarrow{\lambda_{\uparrow}} \cdot \overrightarrow{Q_{\uparrow}}} e^{i \overrightarrow{\lambda_{\downarrow}} \cdot \overrightarrow{Q_{\downarrow}}},
\end{aligned}
$$

where $\chi\left(\overrightarrow{\lambda_{\uparrow}}, \overrightarrow{\lambda_{\downarrow}}\right)$ is its characteristic function that can be expressed within the scattering approach.

For long measurement times $t$, the total characteristic function $\chi$ is the product of contributions from different energies, so that

$$
\chi\left(\overrightarrow{\lambda_{\uparrow}}, \overrightarrow{\lambda_{\downarrow}}\right)=e^{\frac{t}{h} \int d E \log \chi_{E}\left(\overrightarrow{\lambda_{\uparrow}}, \overrightarrow{\lambda_{\downarrow}}\right)}
$$

The energy-resolved characteristic function for the transfer of particles at a given energy $E$ in a structure attached to $n$ leads can be written as [58, 59, 60]

$$
\begin{aligned}
\chi_{E}\left(\overrightarrow{\lambda_{\uparrow}}, \overrightarrow{\lambda_{\downarrow}}\right) & =\left\langle\prod_{j=1, n} e^{i \lambda_{j \uparrow} \hat{N}_{I}^{j \uparrow}} e^{\lambda_{j \downarrow} \hat{N}_{I}^{j \downarrow}}\right. \\
& \left.\times \prod_{j=1, n} e^{-i \lambda_{j \uparrow} \hat{N}_{O}^{j \uparrow}} e^{-i \lambda_{j \downarrow} \hat{N}_{O}^{j \downarrow}}\right\rangle,
\end{aligned}
$$

where the brackets $\langle\ldots\rangle$ stand for the quantum statistical average over the thermal distributions in the leads. Assuming a single channel per lead, $\hat{N}_{O(I)}^{j \sigma}$ is the number operator for outgoing (incoming) particles with spin $\sigma$ in lead $j$ and $\overrightarrow{\lambda_{\uparrow}}, \overrightarrow{\lambda_{\downarrow}}$ are vectors of $n$ real numbers, one for each open channel. In terms of outgoing (incoming) creation operator $\hat{\phi}_{j \sigma}^{\dagger}\left(\hat{a}_{j \sigma}^{\dagger}\right)$, which are linked by the total scattering matrix of the system $S$, the number operators can be expressed as

$$
\hat{N}_{I}^{j \sigma}=\hat{a}_{j \sigma}^{\dagger} \hat{a}_{j \sigma} ; \quad \hat{N}_{O}^{j \sigma}=\hat{\phi}_{j \sigma}^{\dagger} \hat{\phi}_{j \sigma} .
$$

At zero temperature, the statistical average over the Fermi distribution function in Eq. [16] simplifies to the expectation value calculated over the state $|\psi\rangle$ defined in Eq. (8). The interval of integration in Eq. (15) can be separated in two energy ranges, namely $E<\mu$ and $\mu<E<e V$. Since, in the limit of a small voltage bias $V$, $\chi_{E}$ is energy-independent, Eq. (15) can be approximated to

$$
\chi\left(\overrightarrow{\lambda_{\uparrow}}, \overrightarrow{\lambda_{\downarrow}}\right) \simeq\left[\chi_{0}^{\mathrm{S}}\left(\overrightarrow{\lambda_{\uparrow}}, \overrightarrow{\lambda_{\downarrow}}\right)\right]^{M_{\mu}}\left[\chi_{0}^{\mathrm{B}}\left(\overrightarrow{\lambda_{\uparrow}}, \overrightarrow{\lambda_{\downarrow}}\right)\right]^{M-M_{\mu}}
$$

where $M_{\mu}=\mu t / h$ and $M=e V t / h$. 
According to Eq. (14), both single terminal and joint probability distributions require the computation of multidimensional integrals, which can only be performed numerically. In Appendix $\mathrm{C}$ it is shown that the various probability distributions needed to evaluate the $\mathrm{CH}$ inequality can be expressed in a differential form, more suitable for numerical evaluation. All the expectation values needed for the calculations are collected in Appendix $[\mathrm{B}$ Since the two wires are decoupled and there are no spin-flip processes, the joint probabilities with a single analyzer are factorized:

$$
\begin{aligned}
& P^{\theta_{1},-}\left(Q_{1}, Q_{2}\right)=P^{\theta_{1}}\left(Q_{1}\right) P\left(Q_{2}\right), \\
& P^{-, \theta_{2}}\left(Q_{1}, Q_{2}\right)=P\left(Q_{1}\right) P^{\theta_{2}}\left(Q_{2}\right) .
\end{aligned}
$$

Rotational invariance makes $P^{\theta_{1},-}\left(Q_{1}, Q_{2}\right)$ and $P^{-, \theta_{2}}\left(Q_{1}, Q_{2}\right)$ independent of the angle of the analyzers, while $P^{\theta_{1}, \theta_{2}}\left(Q_{1}, Q_{2}\right)$ depends on the angles only through the combination $\frac{\theta_{1} \pm \theta_{2}}{2}$ (upper sign for triplet and lower sign for singlet), so that we can define $P_{1,2}^{\frac{\theta_{1} \pm \theta_{2}}{2}}\left(Q_{1}, Q_{2}\right) \equiv$ $P^{\theta_{1}, \theta_{2}}\left(Q_{1}, Q_{2}\right)$ and $P_{1,-}\left(Q_{1}, Q_{2}\right) \equiv P^{\theta_{1},-}\left(Q_{1}, Q_{2}\right)$. As a result, the $\mathrm{CH}$ inequality depends only on three angles $\theta_{a} \equiv \theta_{1} \pm \theta_{2}, \theta_{b} \equiv \theta_{2} \pm \theta_{1}^{\prime}$ and $\theta_{c} \equiv \theta_{1}^{\prime} \pm \theta_{2}^{\prime}\left(\theta_{d}=\theta_{1} \pm \theta_{2}^{\prime}\right.$ is a linear combination of the other three: $\left.\theta_{d}=\theta_{a}+\theta_{b}+\theta_{c}\right)$. Since $P^{\theta_{1}, \theta_{2}}\left(Q_{1}, Q_{2}\right)$ is an even function of $\frac{\theta_{1} \pm \theta_{2}}{2}$, in order to find maximal violations we can restrict the evaluation of the $\mathrm{CH}$ inequality to the following set of angles: $\theta_{a}=\theta_{b}=\theta_{c}=\theta_{d} / 3 \equiv 2 \Theta$. (This is found by imposing that positive contributions to $\mathcal{S}_{\mathrm{CH}}$ are maximum while negative contributions are minimum.) The quantity $\mathcal{S}_{\mathrm{CH}}$, characterizing the $\mathrm{CH}$ inequality, will therefore depend on a single angle $\Theta$, on the decoherence strength $\alpha$ and on the value of the transmitted charge $Q_{1}$ and $Q_{2}$. As a result, the $\mathrm{CH}$ inequality takes the simplified form

$$
\begin{aligned}
\mathcal{S}_{\mathrm{CH}}= & 3 P_{1,2}^{\Theta}\left(Q_{1}, Q_{2}\right)-P_{1,2}^{3 \Theta}\left(Q_{1}, Q_{2}\right)-P_{1,-}\left(Q_{1}, Q_{2}\right) \\
& -P_{-, 2}\left(Q_{1}, Q_{2}\right) \leq 0 .
\end{aligned}
$$

Without loss of generality we can choose $\sigma=\sigma^{\prime}=\uparrow$. The other cases can be recovered by rotating the polarizers an angle $\pi$.

\section{A. No-enhancement assumption}

As mentioned above, the $\mathrm{CH}$ inequality can be derived under the no-enhancement assumption, Eq. (13). Such a condition is trivially true when a single particle is transmitted, $Q=1$ : The presence of an analyzer can only decrease the counting probability 37 . However, when many particles are transmitted, $Q>1$, the noenhancement assumption is a relationship between distribution probabilities that is not, in general, satisfied for all values of $Q$.

We remind that in the absence of decoherence [35], for given $M$ and $Q$, the no-enhancement assumption in one of the two leads is satisfied only within a range of values of $T$ below certain threshold $T_{\max }(M, Q)$. In the

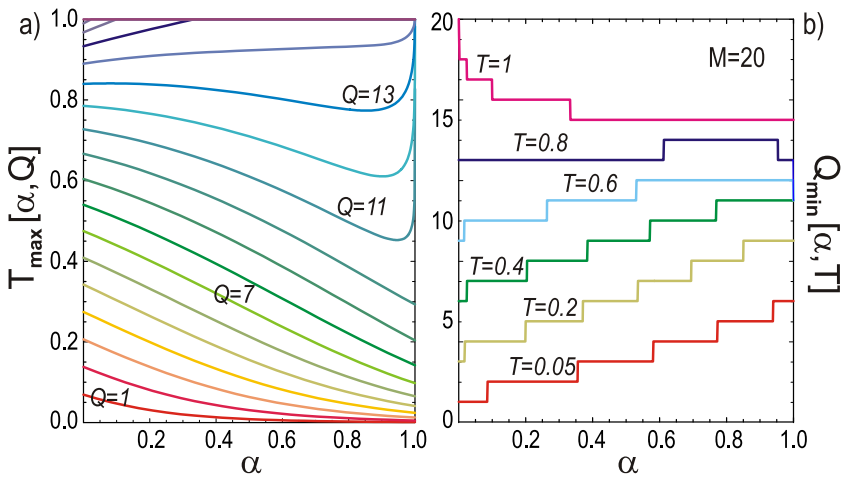

FIG. 2: (Color online) a) Maximum value of the transmission, $T_{\max }$, allowed by the no-enhancement assumption as a function of decoherence rate $\alpha$ for $M=20$ emitted pairs and for the different values of $Q$. b) Minimum allowed number of transmitted particles $Q_{\min }$ for a fixed wire transmission as a function of the decoherence rate.

case of different numbers of transmitted particles in lead 1 and $2\left(Q_{1} \neq Q_{2}\right)$, the maximum allowed transmission probability must be taken to be the minimum between $T_{\max }\left(M, Q_{1}\right)$ and $T_{\max }\left(M, Q_{2}\right)$, according to our assumption of identical wires.

The no-enhancement assumption is affected by decoherence as a consequence of the fact that single terminal probabilities, with or without analyzer, depend on $\alpha$. More precisely, the no-enhancement assumption in one of the two leads is satisfied for transmissions up to a threshold value which is now a function of $\alpha: T_{\max }(\alpha, M, Q)$. Unlike the ideal case, for $\alpha \neq 0$ it is not possible to find an analytical expression for $T_{\max }$. In Fig. 2 $2 T_{\max }$ is plotted as a function of $\alpha$ for $M=20$ and all values of $Q$ from 1 to 20 . One can see that $T_{\max }$ monotonically decreases with $\alpha$ for values of $Q \lesssim M / 2$ and monotonically increases for large values of $Q$. For intermediate values of $Q, T_{\max }$ decreases up to values of $\alpha$ close to one and then rapidly increases reaching one when $\alpha=1$. This behavior is specific of the fictitious lead model and reflects the fact that both the average total current [Eq. [11]], related to $P(Q)$, and the average spin-polarized current, related to $P^{\theta}(Q)$, are increasing functions of $\alpha$. Indeed, as a consequence of a finite $\alpha$, the two distributions shift to larger values of $Q$, as it would happen for an enhanced effective transmission probability. Its maximum allowed value by the no enhancement assumption is therefore reached for a smaller $T$. As a consequence $T_{\max }$ must decrease with $\alpha$. This argument is not valid when $T_{\max } \simeq 1$ at $\alpha=0$, since the average currents do not change appreciably with $\alpha$ and only the peculiar shape of the distributions matters. We define

$$
\begin{aligned}
& T_{\max }\left(\alpha, M, Q_{1}, Q_{2}\right) \\
& =\operatorname{Min}\left[T_{\max }\left(\alpha, M, Q_{1}\right), T_{\max }\left(\alpha, M, Q_{2}\right)\right] .
\end{aligned}
$$

Alternatively, given a wire with a fixed transmission $T$, the no-enhancement assumption is verified for values of $Q$ bigger than or equal to a certain value $Q_{\min }(\alpha, M, T)$. 


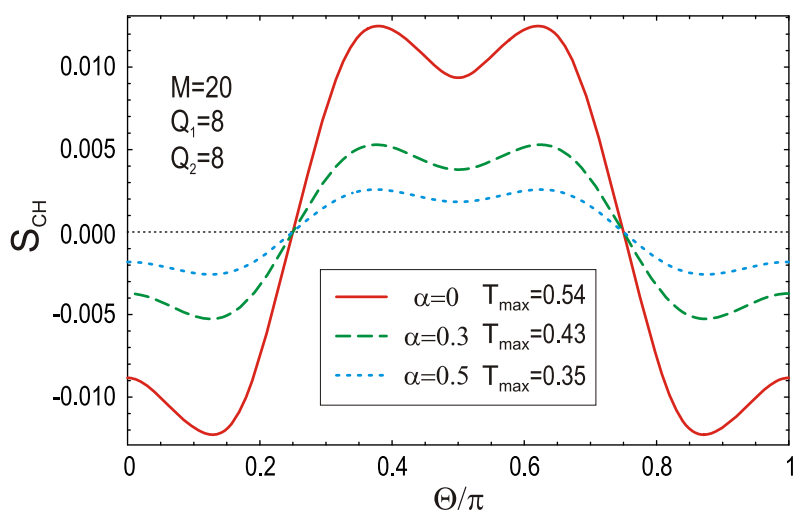

FIG. 3: (Color online) The quantity $\mathcal{S}_{\mathrm{CH}}$ is plotted as a function of $\Theta$ for $M=20, Q_{1}=Q_{2}=8$ and different decoherence rates at the corresponding maximum allowed transmissions. In particular, for $\alpha=0$ (solid line) $T_{\max }=0.54$, for $\alpha=0.3$ (dashed line) $T_{\max }=0.43$ and for $\alpha=0.5$ (dotted line) $T_{\max }=0.35$. The amount of violation of the $\mathrm{CH}$ inequality decreases with $\alpha$, whereas the range of angles for which violation occurs does not change. We call $\Theta_{\text {best }}$ the angle corresponding to the maximum violation.

For $\alpha \neq 0$, the behavior of $Q_{\min }$ is shown in Fig. $2 \mathrm{~b}$ for $M=20$ and for different transmissions. We observe that it increases (step-wise, since only integer values of the number of particles are permitted) as a function of the decoherence rate for almost every transmission $T$, except for those close to unity, for which it decreases. The behavior for small values of $T$ can still be understood in terms of the average current increase with $\alpha$. For $T=1$, being $Q_{\min }=M$ at $\alpha=0$, decoherence can only cause a decrease.

\section{RESULTS}

In the present section we shall discuss how the $\mathrm{CH}$ inequality of Eq. 20] is affected by the presence of decoherence. There are some general characteristics of the behavior of $\mathcal{S}_{\mathrm{CH}}$ that were already found in the absence of decoherence [35] that hold also for finite $\alpha$ [61]. The most relevant are the following:

- $\mathcal{S}_{\mathrm{CH}}$ is always symmetric as a function of $\Theta$ around $\Theta=\pi / 2$

- for given $M, Q_{1}$ and $Q_{2}$ the maximum violation always occurs for $T=T_{\max }\left(\alpha, M, Q_{1}, Q_{2}\right)$.

In Fig. 3 $\mathcal{S}_{\mathrm{CH}}$ is plotted as a function of the angle $\Theta$ for $M=20$ and $Q_{1}=Q_{2}=8$. The three curves refer, respectively, to $\alpha=0$ (solid line), $\alpha=0.3$ (dashed line) and $\alpha=0.5$ (dotted line), each one calculated for the corresponding $T=T_{\max }$ reported in the label box. The plot shows that the $\mathrm{CH}$ inequality is violated within a certain window of values of $\Theta$. The violation is suppressed with increasing decoherence rate, but occurs for the same

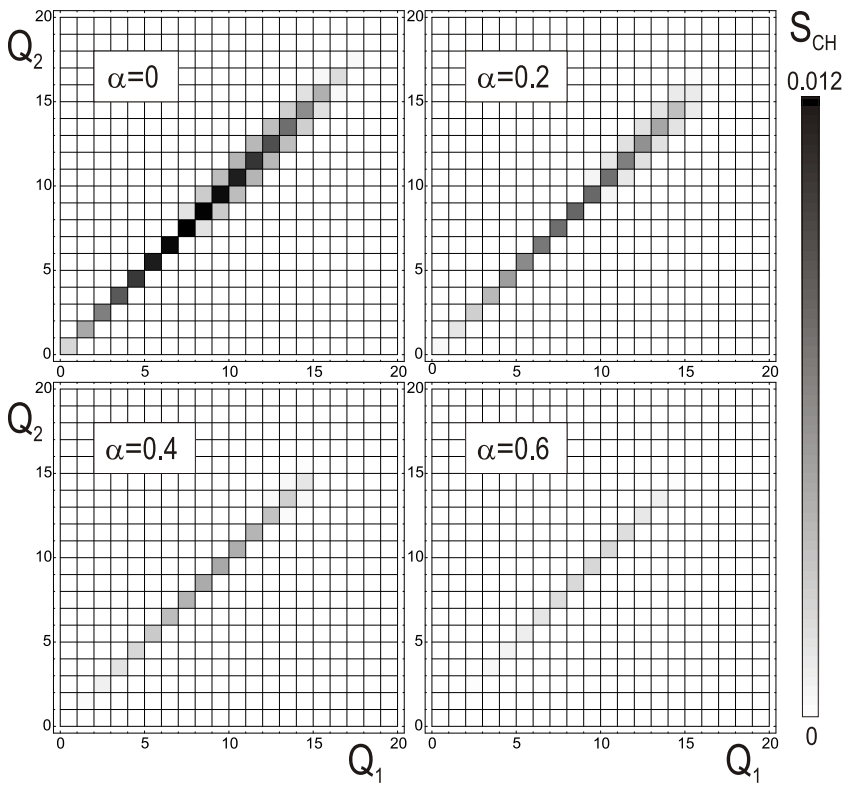

FIG. 4: Density plots of the maximum value of $\mathcal{S}_{\mathrm{CH}}$, evaluated at $T=T_{\max }\left(\alpha, Q_{1}, Q_{2}\right)$ and $\Theta=\Theta_{\text {best }}\left(\alpha, Q_{1}, Q_{2}\right)$, in the $\left(Q_{1}, Q_{2}\right)$ plane for $M=20$ relative to four different values of decoherence $(\alpha=0,0.2,0.4,0.6)$. $\mathcal{S}_{\mathrm{CH}} \simeq 0.012$ is the maximum violation for $M=20$ found in the absence of decoherence.

range of angles. This is due to the following properties of the joint probabilities, which hold at $T=T_{\max }$ for all values of $\alpha$ : i) $P_{1,2}^{\pi / 4}\left(Q_{1}, Q_{2}\right)=P_{1,2}^{3 \pi / 4}\left(Q_{1}, Q_{2}\right)=$ $P_{1,-}\left(Q_{1}, Q_{2}\right)$, as a consequence $\mathcal{S}_{\mathrm{CH}}(\Theta=\pi / 4,3 \pi / 4)=$ 0 ; and ii) $P_{1,2}^{\Theta}\left(Q_{1}, Q_{2}\right) \geq P_{1,2}^{3 \Theta}\left(Q_{1}, Q_{2}\right), P_{1,-}\left(Q_{1}, Q_{2}\right)$, $P_{-, 2}\left(Q_{1}, Q_{2}\right)$ for $\pi / 4 \leq \Theta \leq 3 \pi / 4$. We checked that by reducing $T$ from $T_{\max }$, but keeping $\alpha$ constant, both the window of angles where violation is present and its amount are decreased. Note that between $\Theta=0$ and $\pi / 2$, there is always an angle for which $\mathcal{S}_{\mathrm{CH}}$ is maximum, we shall denote it by $\Theta_{\text {best }}\left(\alpha, M, Q_{1}, Q_{2}\right)$. For given $\alpha$, $M, Q_{1}$ and $Q_{2}$, the maximum violation occurs at $T_{\max }$ and $\Theta_{\text {best }}$.

We now analyze the maximum violation of the $\mathrm{CH}$ inequality for a given $M$ with $T=T_{\max }$ and $\Theta=\Theta_{\text {best }}$ as a function of $Q_{1}, Q_{2}$ and $\alpha$. In Fig. 团 we show four density plots of $\mathcal{S}_{\mathrm{CH}}$ in the $\left(Q_{1}, Q_{2}\right)$ plane for different values of decoherence rate and $M=20$. In the gray scale white corresponds to $\mathcal{S}_{\mathrm{CH}}=0$ and black to its maximum value taken in the absence of decoherence. When $\alpha=0$, the $\mathrm{CH}$ inequality is strongly violated for diagonal terms of the distribution (where $Q_{1}=Q_{2}$ ). However, some weaker violations are also possible for $Q_{1} \neq Q_{2}$, though they tend to disappear with increasing $\alpha$. By increasing the values of $\alpha$ the plots show that the maximum violation of the $\mathrm{CH}$ inequality decreases rapidly: for $\alpha=0.6$ we get only $16 \%$ of the largest value reached at $\alpha=0$. The behavior of the $\mathrm{CH}$ inequality is symmetrical with respect to the exchange of $Q_{1}$ with $Q_{2}$ for any rate of decoherence. In Fig. [5 we report the section of the plots 


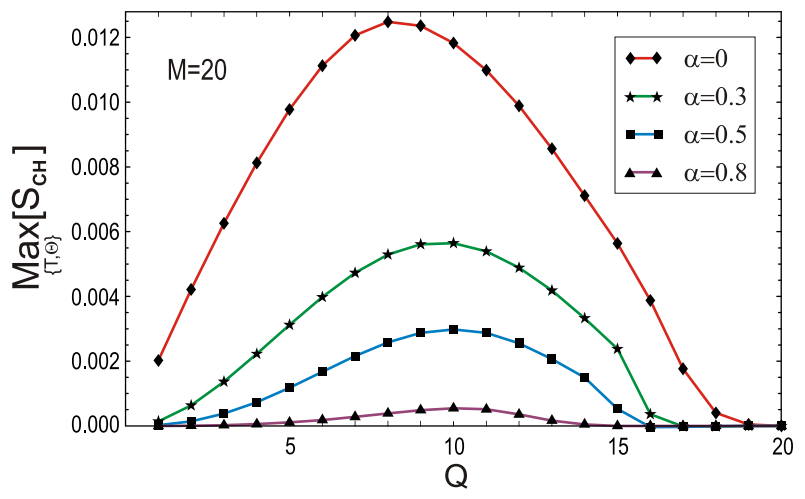

FIG. 5: (Color online) Maximum value of the quantity $\mathcal{S}_{\mathrm{CH}}$ ( $T=T_{\max }$ and $\left.\Theta=\Theta_{\text {best }}\right)$ as a function of $Q$ for $M=20$ and different values of decoherence: $\alpha=0,0.3,0.5,0.8$. The largest violation always occurs in the absence of decoherence and, for a given $Q$, violation is reduced monotonically with $\alpha$. The position where the maximum occurs, $Q_{\text {best }}$, slightly increases with $\alpha$. At $Q=Q_{\text {best }}, T_{\max }=0.54$ for $\alpha=0$, $T_{\max }=0.59$ for $\alpha=0.3, T_{\max }=0.52$ for $\alpha=0.5$ and $T_{\max }=$ 0.39 for $\alpha=0.8$.

in Fig. 4 along the diagonal of the $\left(Q_{1}, Q_{2}\right)$-plane. The four curves are relative to $\alpha=0,0.3,0.5$ and 0.8 an $M=20$. Several observations are in order. If we denote with $Q_{\text {best }}$ the position of the maximum of a curve, for all values of decoherence rate $Q_{\text {best }} \sim M / 2$, more precisely, $Q_{\text {best }}=8$ for $\alpha=0$ and $Q_{\text {best }}=10$ for all other curves. This slight increase of $Q_{\text {best }}$ with $\alpha$ is due to the fact that an increase in decoherence is accompanied by a slight enhancement of the average current [Eq. [11] ] flowing through the wires (as mentioned at the end of Section III). This is, however, a specific feature of the model of decoherence we are considering. Note furthermore that, as decoherence gets stronger, the range of values of $Q$ for which violation takes place shrinks.

We now discuss the violation of the $\mathrm{CH}$ inequality as a function of $\alpha$ and $M$ at $T=T_{\max }$, $\Theta=\Theta_{\text {best }}$ and $Q=Q_{\text {best. }}$. In Fig. [6 the ratio $s \equiv$ $\mathcal{S}_{\mathrm{CH}}\left(\alpha, M, T_{\max }, \Theta_{\text {best }}, Q_{\text {best }}\right) / \mathcal{S}_{\mathrm{CH}}\left(0, M, T_{\max }, \Theta_{\text {best }}, Q_{\text {best }}\right.$ (i. e. the quantity $\mathcal{S}_{\mathrm{CH}}$ normalized to its value in the absence of decoherence) is reported in a three-dimensional plot as a function of $\alpha$ and the number of emitted pairs $M$. The most interesting feature is that such a ratio decays more rapidly with $\alpha$ as $M$ is increased. This means that decoherence is more disruptive, as far as detection of entanglement is concerned, for long measuring times (i.e. large $M$ ). As an example, for $M=1000$ the extent of the violation is reduced by $80 \%$ at $\alpha=0.1$. More precisely, for values of $M$ larger than 30 , we find that the normalized $\mathcal{S}_{\mathrm{CH}}$ follows the law:

$$
s \sim \frac{\sinh \left[K(1-\alpha)^{b \sqrt{M}}\right]}{\sinh (K)},
$$

with $K=7.26$ and $b=0.076$.

Another interesting aspect is related to $Q_{\text {best }}$ which, as mentioned above, only slightly increases with $\alpha$ for

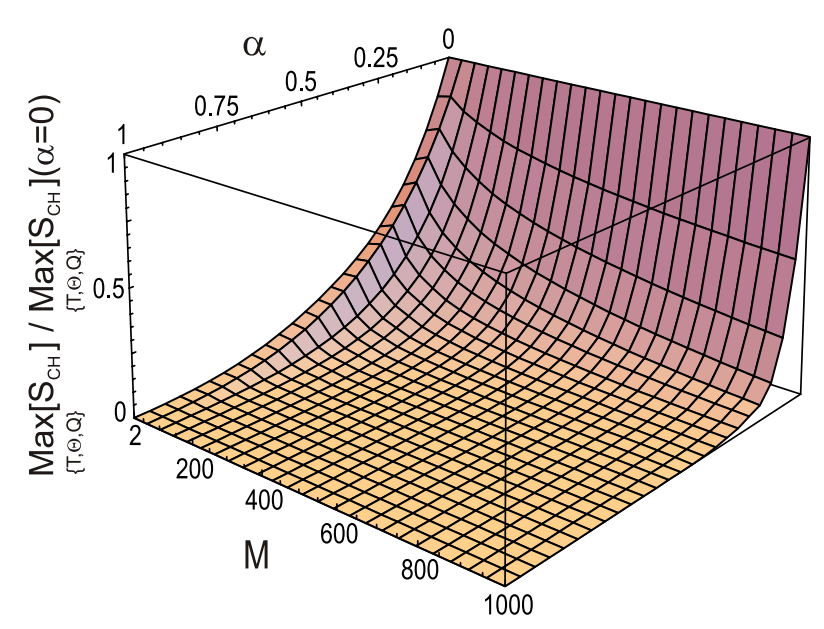

FIG. 6: (Color online) $\mathcal{S}_{\mathrm{CH}}$, normalized to its value in the absence of decoherence and calculated at $T=T_{\max }, \Theta=$ $\Theta_{\text {best }}$ and $Q=Q_{\text {best }}$, is plotted as a function of decoherence rate $\alpha$ and number of injected entangled pairs $M$. Longer measuring times (i.e. larger values of $M$ ) make decoherence more effective, that is, make the detection of entanglement more difficult.

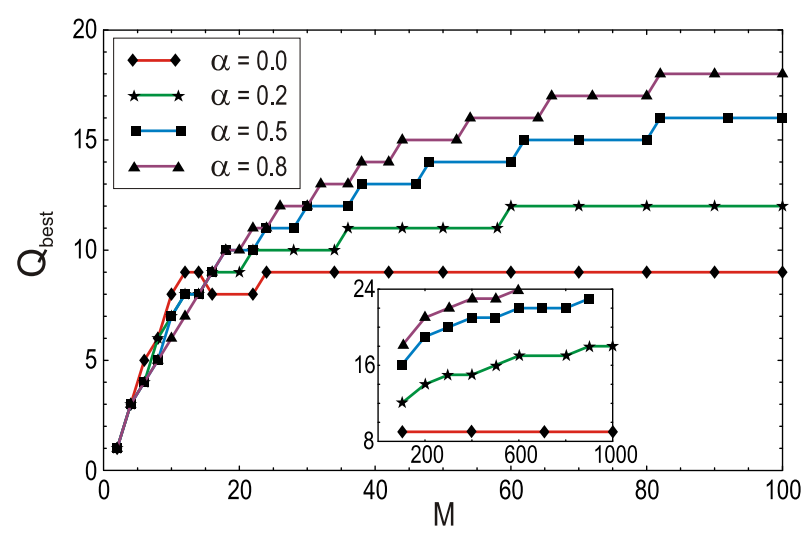

FIG. 7: (Color online) $Q_{\text {best }}$ is plotted as a function of $M$ for different values of decoherence rate $(\alpha=0,0.2,0.5$ and 0.8$)$. In the inset, curves are shown over an extended range, up to $M=1000$. For a given $\alpha$, with increasing $M$ the value of $Q_{\text {best }}$ increases very slowly remaining of the order of 10 .

all values of $M$. As $M$ is increased, the value of $Q_{\text {best }}$, for a given $\alpha$, does not increase proportionally to $M$, but very much slowly and surprisingly remains of the order of 10 for $M=1000$ (see Fig. 17). For $\alpha=0$ this can be understood as follows. On the one hand, one expects $Q_{\text {best }}$, corresponding to the largest $\mathcal{S}_{\mathrm{CH}}$, to be about the position of the maximum of joint probability distributions, which can be assumed to be equal to the product $M T$. On the other hand, $T_{\max }$ is a decreasing function of $M$, in fact it decays as $1 / M[35]$. The product $M T_{\max }$ is therefore expected to be a constant. Indeed, it is possible to show, in the large $M$ expansion, that $Q_{\text {best }} \sim M T_{\max }$ for $\alpha=0$ and $Q_{\text {best }} \sim M \sqrt{T_{\max }}$ for $\alpha \neq 0$, while $T_{\max } \sim 1 / M$ for $\alpha=0$ and $T_{\max } \sim 1 / M^{2}$ 


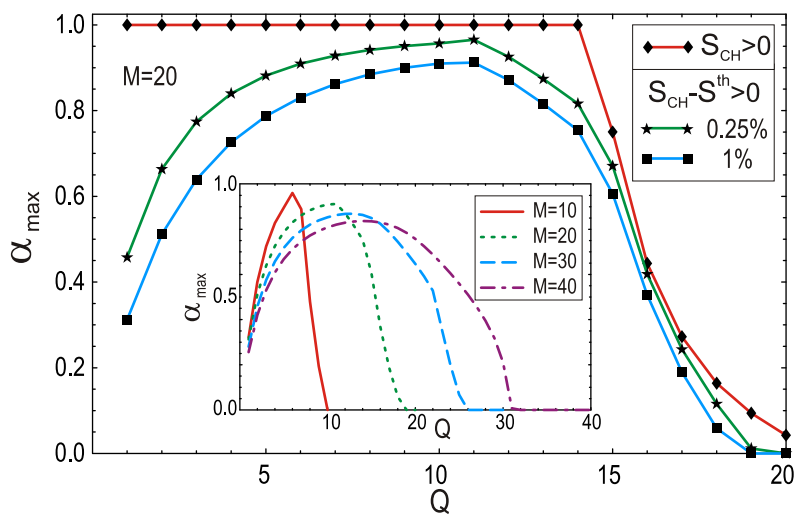

FIG. 8: (Color online) Maximum value $\left(\alpha_{\max }\right)$ of the decoherence parameter for which there is still violation of the $\mathrm{CH}$ inequality as a function of $Q$ for $M=20$. The line with represents $\alpha_{\max }(Q)$ with $\mathcal{S}_{t h}=0$ : from $Q=1$ to $Q=14$ violation is found for any decoherence rate. The line with $\star$ is instead computed using a threshold $\mathcal{S}_{t h}$ which corresponds to $0.25 \%$ of the maximum violation value for $\alpha=0$, and the line with using $\mathcal{S}_{t h}$ corresponding to $1 \%$. The latter threshold is used in the inset where $\alpha_{\max }(Q)$ is plotted for $M=10,20$, 30 and 40 . Interestingly we found that there is a value $\bar{Q}$ that is more robust against decoherence. In particular, $\bar{Q}=6$ for $M=10, \bar{Q}=11$ for $M=20, \bar{Q}=13$ for $M=30$ and $\bar{Q}=14$ for $M=40$. With increasing $M, \alpha_{\max }$ diminishes slowly.

for $\alpha \neq 0$. As a result, $Q_{\text {best }}$ is roughly constant as a function of $M$ and $\alpha$ 62.

The final point we address is the maximum decoherence rate, that we denote by $\alpha_{\max }$, for which there is still violation of the $\mathrm{CH}$ inequality as a function of $Q \equiv Q_{1}=Q_{2}$. In Fig. 8 we plot $\alpha_{\max }$ as a function of $Q$ for $M=20$ at $T=T_{\max }$ and $\Theta=\Theta_{\text {best. }}$. The line with the symbol shows that violation of the $\mathrm{CH}$ inequality is found for any rate of decoherence for $Q=1$ to $Q=14$ and thereafter $\alpha_{\max }$ decreases sharply. Nevertheless, the extent of violation for $\alpha$ close to 1 is almost negligible for most of $1 \leq Q \leq 14$. One can therefore introduce a small positive threshold $\mathcal{S}_{\text {th }}$ which defines the violation as: $\mathcal{S}_{\mathrm{CH}}<\mathcal{S}_{\text {th }}$. The line with $\star$ refers to a threshold of $0.25 \%$ of the maximum value of $\mathcal{S}_{\mathrm{CH}}$ at $\alpha=0\left(\mathcal{S}_{\text {th }}=3 \times 10^{-5}\right.$ for $\left.M=20\right)$, and the line with to a $1 \%\left(\mathcal{S}_{\text {th }}=1.2 \times 10^{-4}\right.$ for $\left.M=20\right)$. The latter percentage is used for the thresholds of the plots in the inset of Fig. 8 where $\alpha_{\max }$ is plotted for $M=10,20,30$ and 40. It is shown that there are values of $Q$ that are more resistent to decoherence, i. e. for which violation survives for larger decoherence rates. In the caption of Fig. 8 the most protected value against decoherence is denoted with $\bar{Q} \equiv Q_{\text {best }}\left(\alpha_{\max }\right)$.

\section{CONCLUSIONS}

In this paper we have studied the effect of decoherence on the violation of the $\mathrm{CH}$ inequality formulated in terms of the FCS [35]. The system under investiga- tion (Fig. 1) consists of an idealized entangler connected, through a pair of identical mesoscopic wires, to spinselective counters. We have assumed that decoherence, which occurs equally but independently in the two conductors, is produced by the presence of additional fictitious reservoirs according to the phenomenological model of Büttiker [48, 49]. Decoherence is parameterized by the rate $\alpha$.

As expected, decoherence gives rise to suppression of the violation of the $\mathrm{CH}$ inequality. The extent of such a suppression has been analyzed as a function of the parameters which characterize the system, namely the transmission $T$ of the wires, the angle between analyzers $\Theta$, the number of injected entangled pairs $M$ and the number of transmitted particles $Q_{1}$ and $Q_{2}$ in the counters. First we have discussed the no-enhancement assumption, a condition that needs to be satisfied in both leads 1 and 2 in order for the $\mathrm{CH}$ inequality to hold. We have found that such condition, in a given lead, is verified for all transmission $T$ up to some maximum value $T_{\max }$ which depends on $Q, M$ and, of course, $\alpha$. In particular, $T_{\max }$ decreases with the decoherence rate up to some value of $Q$ and thereafter increases. The main results can be summarized as follows:

- Maximal violation, even in the presence of decoherence, occurs at the largest allowed transmission $T=T_{\max }$ and for $Q_{1}=Q_{2}$ (it disappears very rapidly when $\left.Q_{1} \neq Q_{2}\right)$.

- As long as $T=T_{\max }$, the angle range of the analyzers for which violation takes place does not depend on the decoherence rate, though the extent of violation decreases with $\alpha$.

- In the absence of decoherence, the maximum violation of the $\mathrm{CH}$ inequality was proved to decay as $1 / M[35]$. Here we have found that, for finite $\alpha$, the parameter $\mathcal{S}_{\mathrm{CH}}$ decreases exponentially with $\sqrt{M}$, more precisely as $[f(\alpha)]^{\sqrt{M}} / M$, i.e. decays both with increasing $M$ and $\alpha$.

- The value of $Q$ for which maximum violation occurs is virtually independent of $M$, which means that the largest violations appear for relatively small numbers of transmitted particles, even at large observation times.

- Interestingly, we have found that the largest decoherence rate for which the $\mathrm{CH}$ inequality is violated (within a given small tolerance) presents a maximum as a function of $Q$. This means that there exist numbers of transmitted charges which are more protected against decoherence, i.e. the influence of the environment is less disruptive as far as the violation of $\mathrm{CH}$ inequality is concerned.

Although, in this paper, dephasing is assumed to be produced by the presence of additional reservoirs, other 
a)
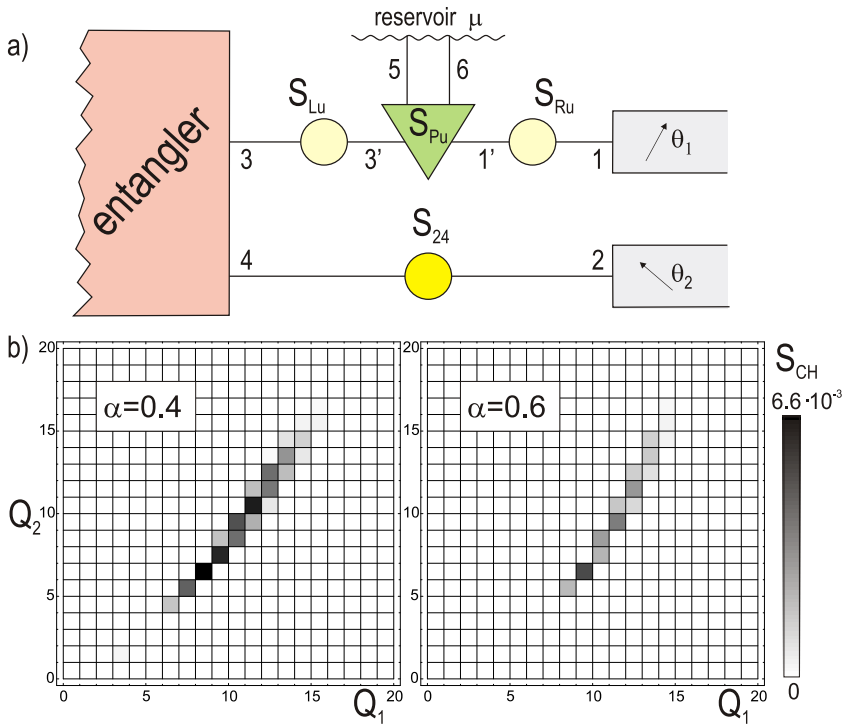

FIG. 9: (Color online) a) Idealized setup with a single additional reservoir in the upper branch of the system. Scattering matrices are chosen so that, in the absence of decoherence, the two conductors have equal transmission. b) Density plot of the maximum value of $\mathcal{S}_{\mathrm{CH}}$ in the $\left(Q_{1}, Q_{2}\right)$ plane for $M=20$ and for $\alpha=0.4$ (left) and $\alpha=0.6$ (right). As decoherence increases, the maximum violation is not achieved on the diagonal, but is shifted towards the right-bottom part of the plane $\left(Q_{1}>Q_{2}\right)$. This occurs because only the current flowing through the conductor affected by decoherence is modified. Furthermore, the suppression of the violation by $\alpha$ is less pronounced with respect to the case with two additional reservoirs. For example, for $\alpha=0$ we find $\operatorname{Max}\left[\mathcal{S}_{\mathrm{CH}}\right]=0.012$ achieved at $\left(Q_{1}=8, Q_{2}=8\right)$. For $\alpha=0.2$ and one additional reservoir we have $\operatorname{Max}\left[\mathcal{S}_{\mathrm{CH}}\right]=0.0089$ reached at $(8,7)$, whereas for two additional reservoirs we get $\operatorname{Max}\left[\mathcal{S}_{\mathrm{CH}}\right]=0.0074$ at $(9,9)$. For $\alpha=0.4$ and one additional reservoir, $\operatorname{Max}\left[\mathcal{S}_{\mathrm{CH}}\right]=0.0066$ at $(9,7)$, and with two additional reservoirs, $\operatorname{Max}\left[\mathcal{S}_{\mathrm{CH}}\right]=0.0042$ at $(10,10)$. Finally, for $\alpha=0.6$ and one additional reservoir, $\operatorname{Max}\left[\mathcal{S}_{\mathrm{CH}}\right]=0.0046$ at $(10,7)$, and with two additional reservoirs, $\operatorname{Max}\left[\mathcal{S}_{\mathrm{CH}}\right]=0.0020$ at $(10,10)$.

different sources of decoherence are possible in mesoscopic systems. We believe that this model captures the main effects of decoherence, as far as violations of the $\mathrm{CH}$ inequality in a mesoscopic system is concerned, and that the results found in this work may be useful to design the best experimental conditions.

Since real systems cannot be perfectly shielded from the environment, the issues analyzed in this work seem adequate not only from a fundamental point of view, but also in what it might contribute to the understanding of the properties of lossy quantum channels. For the future it would be interesting to apply our method to realistic systems, like normal or superconducting beam splitters. Of interest would also be the combined effects of the presence of spin-flipping processes and decoherence.

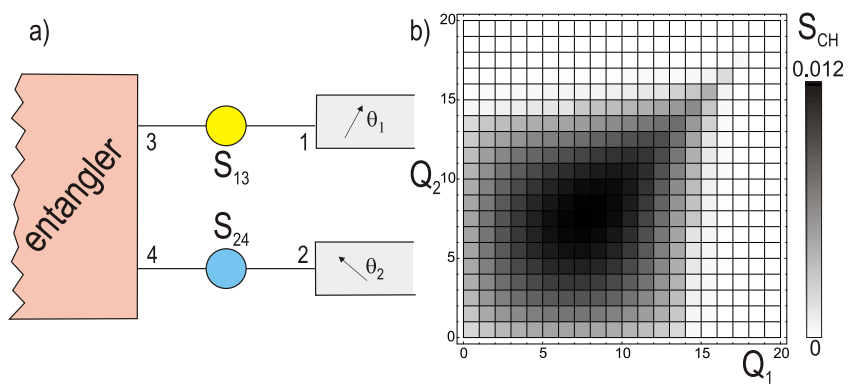

FIG. 10: (Color online) a) Idealized setup with no decoherence but differently transmitting upper and lower conductors. b) The density plot of the maximum value of the quantity $\mathcal{S}_{\mathrm{CH}}$ is shown in the $\left(Q_{1}, Q_{2}\right)$-plane for $M=20$.

\section{Acknowledgments}

The authors would like to thank F. Sols for encouraging this work, P. San-Jose for useful discussions and M. Büttiker and C.W.J. Beenakker for comments on the manuscript. This work was supported by the FPI program of the Comunidad Autónoma de Madrid, the EU under the Marie Curie Research Training Network, EC-RTN Nano, EC-RTN Spintronics and EC-ISTSQUIBIT2.

\section{APPENDIX A: ASYMMETRIC SETUP: ONE ADDITIONAL RESERVOIR}

It is interesting to consider the case in which decoherence affects the two wires differently. In this appendix we study the case when decoherence affects only one of the two conductors, i.e. in the presence of a single additional probe, for example, in the upper branch, as depicted in Fig. 9]. Being $T_{0}$ the transmission of the elastic scatterers in the upper conductor, we choose the transmission of the lower conductor to be equal to $T=T_{0}^{2} /\left(2-T_{0}\right)^{2}$, in order for the two conductors to have the same conductance in the absence of decoherence. Fig. 9] shows the density plot of the maximum value of $S_{\mathrm{CH}}$ (with $T=T_{\max }$ and $\left.\Theta=\Theta_{\text {best }}\right)$ as a function of $Q_{1}$ and $Q_{2}$, for $M=20, \alpha=0.4$ (left) and $\alpha=0.6$ (right). In the presence of decoherence the maximum violation is not achieved on the diagonal $\left(Q_{1}=Q_{2}\right)$, i.e. the behavior of $S_{\mathrm{CH}}$ is not symmetrical anymore with respect to $Q_{1}$ and $Q_{2}$. This is due to the fact that, as we have seen above, the overall current increases with $\alpha$ so that it is more likely to transmit a larger number of particles in the conductor subjected to decoherence. Another difference with respect to the case with two additional reservoirs is that the suppression of the violation by $\alpha$ is less pronounced.

The asymmetry found in the behavior of the density plots of Fig. 9] must not be confused with the asymmetry we would obtain in a setup without decoherence but with conductors of different resistance (system sketched 
in Fig. 10 ). In this case, by applying separately the no enhancement assumption to the two conductors, large violations occur in a vast region of the $\left(Q_{1}, Q_{2}\right)$-plane, as shown in Fig. 10p. Interestingly, we note that one would get large violations of the $\mathrm{CH}$ inequality for $Q_{1} \neq Q_{2}$. However, this asymmetry would not come from the fact that entanglement is weakened by dephasing in only one wire.

\section{APPENDIX B: EXPECTATION VALUES}

The most general expression for the characteristic function when spin- $\sigma$ electrons are counted in lead 1 and spin- $\sigma^{\prime}$ electrons are counted in lead 2 is

$$
\chi_{E}\left(\lambda_{1 \sigma}, \lambda_{2 \sigma^{\prime}}\right)=1+\left(e^{-i \lambda_{1 \sigma}}-1\right)\left\langle\hat{N}_{\mathrm{O}}^{1 \sigma}\right\rangle+\left(e^{-i \lambda_{2 \sigma^{\prime}}}-1\right)\left\langle\hat{N}_{\mathrm{O}}^{2 \sigma^{\prime}}\right\rangle+\left(e^{-i \lambda_{1 \sigma}}-1\right)\left(e^{-i \lambda_{2 \sigma^{\prime}}}-1\right)\left\langle\hat{N}_{\mathrm{O}}^{1 \sigma} \hat{N}_{\mathrm{O}}^{2 \sigma^{\prime}}\right\rangle
$$

for each relevant energy range: $0<E<\mu$ and $\mu<E<e V$. When both spin species are counted in one of the terminals, the characteristic function reads

$$
\begin{aligned}
\chi_{E}\left(\lambda_{1}, \lambda_{2 \sigma^{\prime}}\right)= & 1+\left(e^{-i \lambda_{1}}-1\right)\left\langle\left(\hat{N}_{\mathrm{O}}^{1 \uparrow}+\hat{N}_{\mathrm{O}}^{1 \downarrow}\right)\right\rangle+\left(e^{-i \lambda_{2 \sigma^{\prime}}}-1\right)\left\langle\hat{N}_{\mathrm{O}}^{2 \sigma^{\prime}}\right\rangle+\left(e^{-i \lambda_{1}}-1\right)\left(e^{-i \lambda_{2 \sigma^{\prime}}}-1\right)\left\langle\left(\hat{N}_{\mathrm{O}}^{1 \uparrow}+\hat{N}_{\mathrm{O}}^{1 \downarrow}\right) \hat{N}_{\mathrm{O}}^{2 \sigma^{\prime}}\right\rangle \\
& +\left(e^{-i \lambda_{1}}-1\right)^{2}\left\langle\hat{N}_{\mathrm{O}}^{1 \uparrow} \hat{N}_{\mathrm{O}}^{1 \downarrow}\right\rangle+\left(e^{-i \lambda_{1}}-1\right)^{2}\left(e^{-i \lambda_{2 \sigma^{\prime}}}-1\right)\left\langle\hat{N}_{\mathrm{O}}^{1 \uparrow} \hat{N}_{\mathrm{O}}^{1 \downarrow} \hat{N}_{\mathrm{O}}^{2 \sigma^{\prime}}\right\rangle
\end{aligned}
$$

for counting both spins in terminal 1 , where we have set $\lambda_{1 \uparrow}=\lambda_{1 \downarrow} \equiv \lambda_{1}$.

Using Eqs. (14), (18) and (B1), at zero temperature, one can calculate the single terminal probability distribution:

$$
P^{\theta_{1}}\left(Q_{1 \sigma}\right)=\frac{1}{2 \pi} \int_{-\pi}^{\pi} d \lambda_{1 \sigma}\left[1+\left(e^{-i \lambda_{1 \sigma}}-1\right)\left\langle\hat{N}_{\mathrm{O}}^{1 \sigma}\right\rangle_{S}\right]^{M_{\mu}}\left[1+\left(e^{-i \lambda_{1 \sigma}}-1\right)\left\langle\hat{N}_{\mathrm{O}}^{1 \sigma}\right\rangle_{B}\right]^{M-M_{\mu}} e^{i \lambda_{1 \sigma} Q_{1 \sigma}}
$$

where $\left\langle\hat{N}_{\mathrm{O}}^{1 \sigma}\right\rangle_{S, B} \equiv\left\langle\psi_{S, B}\left|\hat{N}_{\mathrm{O}}^{1 \sigma}\right| \psi_{S, B}\right\rangle$. After integration over $\lambda_{1 \sigma}$ we get

$$
\begin{aligned}
P^{\theta_{1}}\left(Q_{1 \sigma}\right) & =\left(1-\left\langle\hat{N}_{\mathrm{O}}^{1 \sigma}\right\rangle_{S}\right)^{M_{\mu}}\left(1-\left\langle\hat{N}_{\mathrm{O}}^{1 \sigma}\right\rangle_{B}\right)^{M-M_{\mu}-Q_{1 \sigma}}\left(\left\langle\hat{N}_{\mathrm{O}}^{1 \sigma}\right\rangle_{B}\right)^{Q_{1 \sigma}} \\
& \times \sum_{n=\operatorname{Max}\left[0, Q_{1 \sigma}-M+M_{\mu}\right]}^{\operatorname{Min}\left[M_{\mu}, Q_{1 \sigma}\right]}\left(\begin{array}{c}
M_{\mu} \\
n
\end{array}\right)\left(\begin{array}{c}
M-M_{\mu} \\
Q_{1 \sigma}-n
\end{array}\right)\left[\frac{\left\langle\hat{N}_{O}^{1 \sigma}\right\rangle_{S}\left(1-\left\langle\hat{N}_{\mathrm{O}}^{1 \sigma}\right\rangle_{B}\right)}{\left\langle\hat{N}_{\mathrm{O}}^{1 \sigma}\right\rangle_{B}\left(1-\left\langle\hat{N}_{\mathrm{O}}^{1 \sigma}\right\rangle_{S}\right)}\right]^{n}
\end{aligned}
$$

If one chooses $\mu_{\mathrm{L}}=e V$ and $\mu_{\mathrm{R}}=0$, one obtains $\mu=\frac{e V}{2}$ and $M_{\mu}=\frac{M}{2}$. Therefore, in order for $M_{\mu}$ to be integer, $M$ must be an even number.

Using Eqs. (14), (18) and (B2), the single terminal probability distribution when both spin species are counted in the terminal is

$$
\begin{aligned}
& P\left(Q_{1}\right)=\frac{1}{2 \pi} \int_{-\pi}^{\pi}\left[1+\left(e^{-i \lambda_{1}}-1\right)\left\langle\hat{N}_{\mathrm{O}}^{1}\right\rangle_{S}+\left(e^{-i \lambda_{1}}-1\right)^{2}\left\langle\hat{N}_{\mathrm{O}}^{1 \uparrow} \hat{N}_{\mathrm{O}}^{1 \downarrow}\right\rangle_{S}\right]^{M_{\mu}} \\
& \times\left[1+\left(e^{-i \lambda_{1}}-1\right)\left\langle\hat{N}_{\mathrm{O}}^{1}\right\rangle_{B}+\left(e^{-i \lambda_{1}}-1\right)^{2}\left\langle\hat{N}_{\mathrm{O}}^{1 \uparrow} \hat{N}_{\mathrm{O}}^{1 \downarrow}\right\rangle_{B}\right]^{M-M_{\mu}} e^{i \lambda_{1} Q_{1}} d \lambda_{1},
\end{aligned}
$$

where $\left\langle\hat{N}_{\mathrm{O}}^{1}\right\rangle_{S, B} \equiv\left\langle\left(\hat{N}_{\mathrm{O}}^{1 \uparrow}+\hat{N}_{\mathrm{O}}^{1 \downarrow}\right)\right\rangle_{S, B}$.

In the following subsections we collect all the expectation values needed to work out the expressions for the probability distributions of Eq. (20). 


\section{Setup with two additional reservoirs}

Let us consider the setup depicted in Fig. 1 where two additional reservoirs are present. The expectation values for energies $0<E<\mu$ as a function of transmission $T$, decoherence parameter $\alpha$ and analyzers' angle $\Theta$ are

$$
\begin{aligned}
\left\langle\hat{N}_{\mathrm{O}}^{1 \uparrow}\right\rangle_{S} & =\left\langle\hat{N}_{\mathrm{O}}^{2 \uparrow}\right\rangle_{S}=\frac{2 \sqrt{T}\left[\sqrt{T}+\alpha(2-\sqrt{T})-\alpha^{2}(1-\sqrt{T})\right]}{[2-\alpha(1-\sqrt{T})]^{2}}, \\
\left\langle\hat{N}_{\mathrm{O}}^{1}\right\rangle_{S} & =\left\langle\hat{N}_{\mathrm{O}}^{2}\right\rangle_{S}=\frac{4 \sqrt{T}\left[\sqrt{T}+\alpha(2-\sqrt{T})-\alpha^{2}(1-\sqrt{T})\right]}{[2-\alpha(1-\sqrt{T})]^{2}}, \\
\left\langle\hat{N}_{\mathrm{O}}^{1 \uparrow} \hat{N}_{\mathrm{O}}^{1 \downarrow}\right\rangle_{S}=\left\langle\hat{N}_{\mathrm{O}}^{2 \uparrow} \hat{N}_{\mathrm{O}}^{2 \downarrow}\right\rangle_{S}= & \frac{4 T \alpha\left[2 \sqrt{T}+2 \alpha(1-\sqrt{T})-\alpha^{2}(1-\sqrt{T})\right]}{[2-\alpha(1-\sqrt{T})]^{3}}, \\
\left\langle\hat{N}_{\mathrm{O}}^{1 \uparrow} \hat{N}_{\mathrm{O}}^{2 \uparrow}\right\rangle_{S}= & \frac{4 T\left\{\left[\alpha(2-\alpha)+\sqrt{T}\left(1-\alpha+\alpha^{2}\right)\right]^{2}-T(1-\alpha)^{2} \cos \Theta\right\}}{[2-\alpha(1-\sqrt{T})]^{4}}, \\
\left\langle\hat{N}_{\mathrm{O}}^{1} \hat{N}_{\mathrm{O}}^{2 \uparrow}\right\rangle_{S}=\left\langle\hat{N}_{\mathrm{O}}^{1 \uparrow} \hat{N}_{\mathrm{O}}^{2}\right\rangle_{S}= & \frac{8 T\left[\sqrt{T}+\alpha(2-\sqrt{T})-\alpha^{2}(1-\sqrt{T})\right]^{2}}{[2-\alpha(1-\sqrt{T})]^{4}} \\
\left\langle\hat{N}_{\mathrm{O}}^{1 \uparrow} \hat{N}_{\mathrm{O}}^{1 \downarrow} \hat{N}_{\mathrm{O}}^{2 \uparrow}\right\rangle_{S}=\left\langle\hat{N}_{\mathrm{O}}^{1 \uparrow} \hat{N}_{\mathrm{O}}^{2 \uparrow} \hat{N}_{\mathrm{O}}^{2 \downarrow}\right\rangle_{S}= & 8 \sqrt{T^{3}} \alpha\left[2 \sqrt{T}+2 \alpha(1-\sqrt{T})-\alpha^{2}(1-\sqrt{T})\right] \\
& \times \frac{\left[\sqrt{T}+\alpha(2-\sqrt{T})-\alpha^{2}(1-\sqrt{T})\right]}{[2-\alpha(1-\sqrt{T})]^{5}} .
\end{aligned}
$$

The expectation values for energies $\mu<E<e V$ are

$$
\begin{aligned}
\left\langle\hat{N}_{\mathrm{O}}^{1 \uparrow}\right\rangle_{B} & =\left\langle\hat{N}_{\mathrm{O}}^{2 \uparrow}\right\rangle_{B}=\frac{2 T(1-\alpha)}{[2-\alpha(1-\sqrt{T})]^{2}}, \\
\left\langle\hat{N}_{\mathrm{O}}^{1}\right\rangle_{B} & =\left\langle\hat{N}_{\mathrm{O}}^{2}\right\rangle_{B}=\frac{4 T(1-\alpha)}{[2-\alpha(1-\sqrt{T})]^{2}}, \\
\left\langle\hat{N}_{\mathrm{O}}^{1 \uparrow} \hat{N}_{\mathrm{O}}^{1 \downarrow}\right\rangle_{B} & =\left\langle\hat{N}_{\mathrm{O}}^{2 \uparrow} \hat{N}_{\mathrm{O}}^{2 \downarrow}\right\rangle_{B}=0, \\
\left\langle\hat{N}_{\mathrm{O}}^{1 \uparrow} \hat{N}_{\mathrm{O}}^{2 \uparrow}\right\rangle_{B} & =\frac{8 T^{2}(1-\alpha)^{2} \sin ^{2}(\Theta / 2)}{[2-\alpha(1-\sqrt{T})]^{4}}, \\
\left\langle\hat{N}_{\mathrm{O}}^{1} \hat{N}_{\mathrm{O}}^{2 \uparrow}\right\rangle_{B} & =\left\langle\hat{N}_{\mathrm{O}}^{1 \uparrow} \hat{N}_{\mathrm{O}}^{2}\right\rangle_{B}=\frac{8 T^{2}(1-\alpha)^{2}}{[2-\alpha(1-\sqrt{T})]^{4}}, \\
\left\langle\hat{N}_{\mathrm{O}}^{1 \uparrow} \hat{N}_{\mathrm{O}}^{1 \downarrow} \hat{N}_{\mathrm{O}}^{2 \uparrow}\right\rangle_{B} & =\left\langle\hat{N}_{\mathrm{O}}^{1 \uparrow} \hat{N}_{\mathrm{O}}^{2 \uparrow} \hat{N}_{\mathrm{O}}^{2 \downarrow}\right\rangle_{B}=0 .
\end{aligned}
$$

\section{Setup with one additional reservoir}

Let us now consider the asymmetrical setup of Fig. 9] where there is only one additional reservoir. For energies $0<E<\mu$ we have that $\left\langle\hat{N}_{\mathrm{O}}^{1 \uparrow}\right\rangle_{S},\left\langle\hat{N}_{\mathrm{O}}^{1}\right\rangle_{S}$ and $\left\langle\hat{N}_{\mathrm{O}}^{1 \uparrow} \hat{N}_{\mathrm{O}}^{1 \downarrow}\right\rangle_{S}$ are equal to the case with two fictitious reservoirs. The other expectation values are

$$
\begin{aligned}
\left\langle\hat{N}_{\mathrm{O}}^{2 \uparrow}\right\rangle_{S} & =\frac{T}{2}, \\
\left\langle\hat{N}_{\mathrm{O}}^{2}\right\rangle_{S} & =T \\
\left\langle\hat{N}_{\mathrm{O}}^{2 \uparrow} \hat{N}_{\mathrm{O}}^{2 \downarrow}\right\rangle_{S} & =0, \\
\left\langle\hat{N}_{\mathrm{O}}^{1 \uparrow} \hat{N}_{\mathrm{O}}^{2 \uparrow}\right\rangle_{S} & =\frac{\sqrt{T^{3}} \alpha(2-\alpha)+T^{2}\left[1-\alpha+\alpha^{2}-(1-\alpha) \cos \Theta\right]}{[2-\alpha(1-\sqrt{T})]^{2}}, \\
\left\langle\hat{N}_{\mathrm{O}}^{1} \hat{N}_{\mathrm{O}}^{2 \uparrow}\right\rangle_{S} & =\left\langle\hat{N}_{\mathrm{O}}^{1 \uparrow} \hat{N}_{\mathrm{O}}^{2}\right\rangle_{S}=\frac{2 \sqrt{T^{3}}\left[\sqrt{T}+\alpha(2-\sqrt{T})-\alpha^{2}(1-\sqrt{T})\right]}{[2-\alpha(1-\sqrt{T})]^{4}}, \\
\left\langle\hat{N}_{\mathrm{O}}^{1 \uparrow} \hat{N}_{\mathrm{O}}^{1 \downarrow} \hat{N}_{\mathrm{O}}^{2 \uparrow}\right\rangle_{S} & =\frac{2 T^{2} \alpha\left[2 \sqrt{T}+2 \alpha(1-\sqrt{T})-\alpha^{2}(1-\sqrt{T})\right]}{[2-\sqrt{T})]^{3}}, \\
\left\langle\hat{N}_{\mathrm{O}}^{1 \uparrow} \hat{N}_{\mathrm{O}}^{2 \uparrow} \hat{N}_{\mathrm{O}}^{2 \downarrow}\right\rangle_{S} & =0 .
\end{aligned}
$$


For energies $\mu<E<e V$ we have that $\left\langle\hat{N}_{\mathrm{O}}^{1 \uparrow}\right\rangle_{B},\left\langle\hat{N}_{\mathrm{O}}^{1}\right\rangle_{B},\left\langle\hat{N}_{\mathrm{O}}^{1 \uparrow} \hat{N}_{\mathrm{O}}^{1 \downarrow}\right\rangle_{B},\left\langle\hat{N}_{\mathrm{O}}^{2 \uparrow} \hat{N}_{\mathrm{O}}^{2 \downarrow}\right\rangle_{B},\left\langle\hat{N}_{\mathrm{O}}^{1 \uparrow} \hat{N}_{\mathrm{O}}^{1 \downarrow} \hat{N}_{\mathrm{O}}^{2 \uparrow}\right\rangle_{B}$ and $\left\langle\hat{N}_{\mathrm{O}}^{1 \uparrow} \hat{N}_{\mathrm{O}}^{2 \uparrow} \hat{N}_{\mathrm{O}}^{2 \downarrow}\right\rangle_{B}$ are equal to the case with two additional reservoirs. The other expectation values are

$$
\begin{aligned}
\left\langle\hat{N}_{\mathrm{O}}^{2 \uparrow}\right\rangle_{B} & =\frac{T}{2}, \\
\left\langle\hat{N}_{\mathrm{O}}^{2}\right\rangle_{B} & =T \\
\left\langle\hat{N}_{\mathrm{O}}^{1 \uparrow} \hat{N}_{\mathrm{O}}^{2 \uparrow}\right\rangle_{B} & =\frac{2 T^{2}(1-\alpha) \sin ^{2}(\Theta / 2)}{[2-\alpha(1-\sqrt{T})]^{2}}, \\
\left\langle\hat{N}_{\mathrm{O}}^{1} \hat{N}_{\mathrm{O}}^{2 \uparrow}\right\rangle_{B} & =\left\langle\hat{N}_{\mathrm{O}}^{1 \uparrow} \hat{N}_{\mathrm{O}}^{2}\right\rangle_{B}=\frac{2 T^{2}(1-\alpha)}{[2-\alpha(1-\sqrt{T})]^{2}} .
\end{aligned}
$$

\section{APPENDIX C: PROBABILITY DISTRIBUTIONS}

In order to calculate the various probabilities needed to evaluate the $\mathrm{CH}$ inequality, Eq. (12) and (20), it is necessary to solve the integrals of Eq. (14), where the different characteristic functions are given in Eq. (B1) and Eq. (B2). As we mentioned in Section III] explicit expressions of the probability distributions in terms of sums are lengthy and complicated for practical calculations. It is possible, nevertheless, to express the result for the various probabilities in a quite simple fashion, which makes them manageable for computational analysis. The point is to realize that the characteristic functions are nothing but polynomial functions on the variables $e^{i \lambda_{i}}$ of different degrees. The effect of each integral of Eq. (14), together with its accompanying complex exponential $e^{i \lambda_{i} Q_{i}} /(2 \pi)$, is simply to select the coefficient of the characteristic function polynomial which corresponds to the power equal to $Q_{i}$. Consequently, the result of the integrals for the various probabilities can be expressed in terms of $Q_{i}$ order derivatives of the characteristic function, as we show below. Note that, whenever both analyzers are present, we will choose without loss of generality: $\sigma=\uparrow$ and $\sigma^{\prime}=\uparrow$. For single terminal probability distributions we will also set $\sigma=\uparrow$ for lead 1 and $\sigma^{\prime}=\uparrow$ for lead 2 , although resulting expressions will not depend either on the direction of the spin or on the angle of the analyzer.

For the single terminal probability distribution with analyzer we have

$$
P^{\theta_{1}}\left(Q_{1 \uparrow}\right)=\frac{1}{2 \pi} \int_{-\pi}^{\pi} d \lambda_{1 \uparrow} e^{i \lambda_{1 \uparrow} Q_{1 \uparrow}} \chi\left(\lambda_{1 \uparrow}\right)=\left.\frac{1}{Q_{1 \uparrow} !} \frac{d^{Q_{1 \uparrow}} \chi\left(\lambda_{1 \uparrow}\right)}{d\left(e^{i \lambda_{1 \uparrow}}\right)^{Q_{1 \uparrow} \uparrow}}\right|_{e^{i \lambda_{1 \uparrow} \rightarrow 0}},
$$

where $\chi\left(\lambda_{1 \uparrow}\right)$ can be extracted from Eq. (B1) making $\lambda_{2 \uparrow}=0$ and using Eq. (18). Since $\mu=\frac{e V}{2}$, we have that $M_{\mu}=\frac{M}{2}$, being $M$ the total number of emitted particles per lead or per spin. The expectation values needed in Eq. (B1) above and below energy $\mu$ are given in Appendix B. The single terminal probability distribution in the absence of analyzer is

$$
P\left(Q_{1}\right)=\frac{1}{2 \pi} \int_{-\pi}^{\pi} d \lambda_{1} e^{i \lambda_{1} Q_{1}} \chi\left(\lambda_{1}\right)=\left.\frac{1}{Q_{1} !} \frac{d^{Q_{1}} \chi\left(\lambda_{1}\right)}{d\left(e^{i \lambda_{1}}\right)^{Q_{1}}}\right|_{e^{i \lambda_{1} \rightarrow 0}},
$$

where the characteristic function can be extracted now from Eq. [B2), setting again $\lambda_{2 \uparrow}=0$. We can get similarly the expressions for $P^{\theta_{2}}\left(Q_{2 \uparrow}\right)$ and $P\left(Q_{2}\right)$.

The joint probability distribution when both analyzers are present gives

$$
\begin{aligned}
P^{\theta_{1}, \theta_{2}}\left(Q_{1 \uparrow}, Q_{2 \uparrow}\right) & =\frac{1}{(2 \pi)^{2}} \int_{-\pi}^{\pi} d \lambda_{1 \uparrow} e^{i \lambda_{1 \uparrow} Q_{1 \uparrow}} \int_{-\pi}^{\pi} d \lambda_{2 \uparrow} e^{i \lambda_{2 \uparrow} Q_{2 \uparrow}} \chi\left(\lambda_{1 \uparrow}, \lambda_{2 \uparrow}\right) \\
& =\left.\frac{1}{Q_{1 \uparrow} ! Q_{2 \uparrow} !} \frac{d^{Q_{1 \uparrow}} d^{Q_{2 \uparrow}} \chi\left(\lambda_{1 \uparrow}, \lambda_{2 \uparrow}\right)}{d\left(e^{i \lambda_{1 \uparrow}}\right)^{Q_{1 \uparrow}} d\left(e^{i \lambda_{2 \uparrow}}\right)^{Q_{2 \uparrow} \uparrow}}\right|_{e^{i \lambda_{1 \uparrow}}, e^{i \lambda_{2 \uparrow} \uparrow 0}},
\end{aligned}
$$

which only depends on the angle $\Theta \equiv\left(\theta_{1} \pm \theta_{2}\right) / 2$, as we showed in Section 11 When there is only one analyzer we have

$$
P^{\theta_{1},-}\left(Q_{1 \uparrow}, Q_{2}\right)=\frac{1}{(2 \pi)^{2}} \int_{-\pi}^{\pi} d \lambda_{1 \uparrow} e^{i \lambda_{1 \uparrow} Q_{1 \uparrow}} \int_{-\pi}^{\pi} d \lambda_{2} e^{i \lambda_{2} Q_{2}} \chi\left(\lambda_{1 \uparrow}, \lambda_{2}\right)=\left.\frac{1}{Q_{1 \uparrow} ! Q_{2} !} \frac{d^{Q_{1 \uparrow}} d^{Q_{2}} \chi\left(\lambda_{1 \uparrow}, \lambda_{2}\right)}{d\left(e^{i \lambda_{1 \uparrow}}\right)^{Q_{1 \uparrow}} d\left(e^{i \lambda_{2}}\right)^{Q_{2}}}\right|_{e^{i \lambda_{1 \uparrow}}, e^{i \lambda_{2} \rightarrow 0}}
$$

and

$$
P^{-, \theta_{2}}\left(Q_{1}, Q_{2 \uparrow}\right)=\frac{1}{(2 \pi)^{2}} \int_{-\pi}^{\pi} d \lambda_{1} e^{i \lambda_{1} Q_{1}} \int_{-\pi}^{\pi} d \lambda_{2 \uparrow} e^{i \lambda_{2 \uparrow} Q_{2 \uparrow}} \chi\left(\lambda_{1}, \lambda_{2 \uparrow}\right)=\left.\frac{1}{Q_{1} ! Q_{2 \uparrow} !} \frac{d^{Q_{1}} d^{Q_{2 \uparrow}} \chi\left(\lambda_{1}, \lambda_{2 \uparrow}\right)}{d\left(e^{i \lambda_{1}}\right)^{Q_{1}} d\left(e^{i \lambda_{2 \uparrow}}\right)^{Q_{2 \uparrow}}}\right|_{e^{i \lambda_{1}}, e^{i \lambda_{2} \uparrow} \rightarrow 0} .
$$


However, this two last expressions are not strictly needed since one can use the relations in Eq. (19). Again, all the expectation values which are needed for evaluating these probabilities are given in Appendix B]

[1] J.S. Bell, Speakable and unspeakable in Quantum Mechanics (Cambridge University Press, Cambridge, England, 1987).

[2] M.A. Nielsen, and I.L. Chuang, Quantum Computation and Quantum Information (Cambridge University Press, Cambridge, England, 2000).

[3] A. Zeilinger, Rev. Mod. Phys. 71, S288 (1999).

[4] A. Rauschenbeutel, G. Nogues, S. Osnaghi, P. Bertet, M. Brune, J.-M. Raimond, and S. Haroche, Science 288, 2024 (2000).

[5] C.A. Sackett, D. Kielpinski, B.E. King, C. Langer, V. Meyer, C.J. Myatt, M. Rowe, Q.A. Turchette, W.M. Itano, D.J. Wineland, and C. Monroe, Nature (London) 404, 256 (2000).

[6] D. Loss, and D.P. DiVincenzo, Phys. Rev. A 57, 120 (1998).

[7] Yu. Makhlin, G. Schön, and A. Shnirman, Rev. Mod. Phys. 73, 357 (2001).

[8] Semiconductor Spintronics and Quantum Computation, edited by D.D. Awschalom, D. Loss, and N. Samarth, Series on Nanoscience and Technology (Springer-Verlag, Berlin, 2002).

[9] P. Recher, E.V. Sukhorukov, and D. Loss, Phys. Rev. B 63, 165314 (2001)

[10] G.B. Lesovik, T. Martin, and G. Blatter, Eur. Phys. J. B 24, 287 (2001).

[11] N.M. Chtchelkatchev, G. Blatter, G.B. Lesovik, and T. Martin, Phys. Rev. B 66, 161320(R) (2002).

[12] P. Samuelsson, E.V. Sukhorukov, and M. Büttiker, Phys. Rev. Lett. 91, 157002 (2003).

[13] P. Recher, and D. Loss, Phys. Rev. Lett. 91, 267003 (2003)

[14] E. Prada, and F. Sols, Eur. Phys. J. B 40, 379 (2004).

[15] P. Samuelsson, E.V. Sukhorukov, and M. Büttiker, Phys. Rev. B 70, 115330 (2004).

[16] O. Sauret, D. Feinberg, and T. Martin, Phys. Rev. B 70, 245313 (2004).

[17] P. Recher, and D. Loss, Phys. Rev. B 65, 165327 (2002).

[18] C. Bena, S. Vishveshwara, L. Balents, and M.P.A. Fisher, Phys. Rev. Lett. 89, 037901 (2002).

[19] V. Bouchiat, N. Chtchelkatchev, D. Feinberg, G.B. Lesovik, T. Martin, and J. Torres, Nanotechnology 14, 77 (2003).

[20] W.D. Oliver, F. Yamaguchi, and Y. Yamamoto, Phys. Rev. Lett. 88, 037901 (2002).

[21] D.S. Saraga, and D. Loss, Phys. Rev. Lett. 90, 166803 (2003).

[22] C.W.J. Beenakker, M. Kindermann, C.M. Marcus, and A. Yacoby, Fundamental Problems of Mesoscopic Physics, edited by I.V. Lerner, B.L. Altshuler, and Y. Gefen, NATO Science Series II. Vol. 154 (Kluwer, Dordrecht, 2004).

[23] A.T. Costa and S. Bose, Phys. Rev. Lett. 87, 277901 (2001).

[24] C.W.J. Beenakker, C. Emary, M. Kindermann, and J.L. van Velsen, Phys. Rev. Lett. 91, 147901 (2003).

[25] P. Samuelsson, E.V. Sukhorukov, and M. Büttiker, Phys. Rev. Lett. 92, 026805 (2004).

[26] C.W.J. Beenakker, and M. Kindermann, Phys. Rev. Lett. 92, 056801 (2004).

[27] C.W.J. Beenakker, C. Emary, and M. Kindermann, Phys. Rev. B 69, 115320 (2004).

[28] P. Samuelsson, and M. Büttiker, cond-mat/0410581

[29] D.S. Saraga, B.L. Altshuler, D. Loss, and R.M. Westervelt, Phys. Rev. B 71, 045338 (2005).

[30] G. Burkard, D. Loss, and E.V. Sukhorukov, Phys. Rev. B 61, R16303 (2000).

[31] F. Taddei, and R. Fazio, Phys. Rev. B 65, 075317 (2002).

[32] W.K. Wootters, Phys. Rev. Lett. 80, 2245 (1998).

[33] S. Kawabata, J. Phys. Soc. Jpn. 70, 1210 (2001).

[34] O. Sauret and D. Feinberg, Phys. Rev. Lett. 92, 106601 (2004); O. Sauret, T. Martin and D. Feinberg, cond-mat/0410325

[35] L. Faoro, F. Taddei, and R. Fazio, Phys. Rev. B 69, 125326 (2004).

[36] Note that we make use of the $\mathrm{CH}$ inequality as a tool to detect the persistence of entanglement in the presence of decoherence processes, given an injected entangled state (whose existence is assumed).

[37] J.F. Clauser, and M.A. Horne, Phys. Rev. D 10, 526 (1974).

[38] L. Mandel, and E. Wolf, Optical coherence and quantum optics (Cambridge University Press, Cambridge, England, 1995).

[39] Recently in the literature there have been proposals to measure the FCS [J. Tobiska, and Yu. V. Nazarov, Phys. Rev. Lett. 93, 106801 (2004); J. P. Pekola, Phys. Rev. Lett. 93, 206601 (2004)] and a first experiment has been performed (only in the tunnelling limit at present [J. Bylander, T. Duty, and P. Delsing, Nature 434, 361 (2005)]).

[40] P. Samuelsson, E.V. Sukhorukov, and M. Büttiker, Turk. J. Phys. 27, 481 (2003).

[41] J.L. van Velsen, M. Kindermann, and C.W.J. Beenakker, Turk. J. Phys. 27, 323 (2003).

[42] G. Burkard, and D. Loss, Phys. Rev. Lett. 91, 087903 (2003).

[43] G. Seelig, and M. Büttiker, Phys. Rev. B 64, 245313 (2001).

[44] F. Marquardt, and C. Bruder, Phys. Rev. B 70, 125305 (2004).

[45] M.G. Pala, and G. Iannaccone, Phys. Rev. B 69, 235304 (2004).

[46] M.G. Pala, and G. Iannaccone, Phys. Rev. Lett. 93, 256803 (2004).

[47] H. Förster, S. Pilgram, and M. Büttiker, cond-mat/0502400

[48] M. Büttiker, Phys. Rev. B 33, 3020 (1986).

[49] M. Büttiker, IBM J. Res. Dev. 32, 63 (1988).

[50] Very recently an alternative to Büttiker's dephasing model was put forward by Beenakker [51]: the fictitious 
lead is replaced by a fictitious stub. It turns out that voltage fluctuations in the stub suppress quantum interference effects without affecting the noise properties.

[51] C.W.J. Beenakker, and B. Michaelis, cond-mat/0503347

[52] M. Büttiker, Y. Imry, and M.Ya. Azbel, Phys. Rev. A 30, 1982 (1984).

[53] S. Datta, Electronic Transport in Mesoscopic Systems (Cambridge University Press, Cambridge, England, 1995).

[54] C.W.J. Beenakker, and M. Büttiker, Phys. Rev. B 46, R1889 (1992).

[55] Ya.M. Blanter, and M. Büttiker, Phys. Rep. 336, 1 (2000).

[56] A. Brataas, Yu. V. Nazarov, and G. E. W. Bauer, Eur. Phys. J. B 22, 99 (2001).

[57] R. Landauer, IBM J. Res. Dev. 1, 223 (1957); M. Büttiker, Phys. Rev. Lett. 57, 1761 (1986); A. Szafer, and A. D. Stone, IBM J. Res. Dev. 32, 384 (1988).

[58] B.A. Muzykantskii, and D.E. Khmelnitskii, Phys. Rev. B
50, 3982 (1994).

[59] L.S. Levitov, and G.B. Lesovik, Pis'ma Zh. Eksp. Teor. Fiz. 58, 225 (1993) [JETP Lett. 58, 225 (1993)].

[60] L.S. Levitov, H. Lee, and G.B. Lesovik, Jour. Math. Phys. 37, 10 (1996).

[61] In particular, if the entangler is substituted with a source that emits factorized states, the $\mathrm{CH}$ inequality is never violated for any decoherence rate. This is simply due to the fact that two terminal joint probability distributions are given by the product of single terminal probability distributions, thus making the $\mathrm{CH}$ inequality always equal to zero.

[62] More precisely, for large $M, Q_{\text {best }}$ is implicitly given by the equation: $Q_{\text {best }}=\tilde{Q}\left[\alpha, T_{\max }\left(\alpha, M, Q_{\text {best }}\right)\right]$, where $\tilde{Q}[\alpha, T]=M\left[\sqrt{T} \alpha(2-\alpha)+T\left(2-2 \alpha+\alpha^{2}\right)\right] /[2+(\sqrt{T}-$ 1) $\alpha]^{2}$ is the position of the maximum of the distribution in the large $M$ approximation. 